\title{
Sitting comfortably versus lying down: Is there really a difference in energy expenditure?
}

\author{
J.L. Miles-Chan*, D. Sarafian, J.P. Montani, Y. Schutz, A.G. Dulloo** \\ Department of Medicine/Physiology, University of Fribourg, Chemin du Musée 5, CH-1700 Fribourg, Switzerland
}

\begin{abstract}
Background \& aims: Energy expenditure (EE) during sitting is widely assumed to be higher than that while lying down, but supporting evidence is equivocal. Despite this, resting EE in the sitting position is often used as a proxy for basal metabolic rate. Here we investigate whether EE differs in the comfortable seated position compared to supine (lying) position.

Methods: EE and respiratory quotient (RQ) were measured (by ventilated hood indirect calorimetry) in 19 healthy subjects ( 9 men, 10 women) after an overnight fast. Supine measurements were made using a comfortable clinical tilting table and sitting measurements made using an adjustable, ergonomic car seat adapted for the hood system. After about $30 \mathrm{~min}$ of rest in either position, metabolic monitoring was conducted until stabilization of EE for at least $15 \mathrm{~min}$ in each posture.

Results: EE in the sitting position was not significantly different compared to supine ( $<2 \%$ difference). By contrast, heart rate was higher by 7 beats/min $(p<0.05)$. RQ was slightly but significantly decreased during sitting compared to lying $(p<0.05)$, with no change in breathing rate.

Conclusions: This study suggests that the ventilated hood calorimetry system for assessment of REE after an overnight fast in a comfortable sitting position can be used as a good proxy of the basal metabolic rate. It also underscores the applicability of the ventilated hood system to measurements of resting EE in the sitting posture which, compared to supine posture, may be more acceptable/convenient to the subject/ patient participating in postprandial metabolic studies lasting several hours.
\end{abstract}

\section{Introduction}

With the health risks associated with sedentary behavior now firmly established, ${ }^{1}$ and the search underway for a panacea to the surging prevalence of lifestyle-related disease, low-level physical activity has become a burgeoning area of research. Such research is often focused on ways of decreasing time spent sitting or lying in order to increase overall energy expenditure (EE). The key dogma of this work is the notion that different postures (i.e., lying, sitting, standing) vary in terms of energetic cost, and therefore decreasing time spent in "cheaper" postures will increase overall EE and impact on body weight regulation and health. However direct evidence in support of this notion is equivocal. For example, recently

Non-standard abbreviations: EE, energy expenditure; REE, resting energy expenditure; BMR, basal metabolic rate; RQ respiratory quotient; HR, heart rate $\mathrm{BR}$, breathing rate.

* Corresponding author. Tel.: +41 26300 8589; fax: +41263009734.

** Corresponding author. Tel.: +4126300 8624; fax: +41263009734.

E-mail addresses: jenniferlynn.miles@unifr.ch (J.L. Miles-Chan), abdul.dulloo@ unifr.ch (A.G. Dulloo) we have demonstrated that, in many individuals, EE during standing is no different to that of sitting comfortably, ${ }^{2}$ thus begging the question as to whether or not other basic postures, such as lying and sitting, differ appreciably in terms of energetic cost.

To date, few studies have compared EE in the sitting and supine positions after an overnight fast, and those which do present conflicting results. For example, McCarthy, ${ }^{3}$ and more recently Levine et al., ${ }^{4}$ showed very small and insignificant increases of $1.6 \%$ and $3.7 \%$ respectively in the energy cost of sitting compared to lying, whereas Kanade et al. $^{5}$ reported a significant increase in EE of approximately $25 \%$. Furthermore, such studies often assess metabolic rate using techniques that require a face-mask or mouthpiece/ nose clip, which may lead to an under- or overestimation of resting EE (REE), basal metabolic rate (BMR) or both, ${ }^{6}$ or do not adhere to the strictly standardized conditions of BMR measurement.

The investigation of energy requirements and $\mathrm{EE}$ is fundamental to many areas of both basic and clinical research, with BMR and REE being the two most widely reported measures of $\mathrm{EE}$ and which account for more than two-thirds of daily EE in most healthy adults. There is, however, confusion about interchangeability of the terms BMR and REE. BMR is defined as the minimal energy cost of living, 
and measured under strictly standardized conditions; namely, in the post-absorptive state (following 10-12 h overnight fast), thermal comfort, completely at rest, and fully awake. ${ }^{7}$ In contrast, measurements of REE are generally much less standardized between (and often within) experimental settings, in particular the timing of its measurement after the last meal, such that REE could include part of the thermic effects of food and drink and hence overestimate BMR. Additionally, REE is often measured in the sitting position - a posture which may be more comfortable for the subject during long measurements and/or more practical in a variety of experimental settings. This raises the question as to the extent to which REE in the sitting position can be used as a proxy for BMR, under otherwise identical standardized conditions as BMR.

In this context, the objective of the present study was to investigate whether REE measured in a comfortable seated posture would differ from BMR measured in a supine posture, using a ventilated hood indirect calorimetry.

\section{Materials and methods}

\subsection{Subjects}

19 healthy adults ( 9 men, 10 women) were studied with a mean ( \pm SEM) age of $24 \pm 1 \mathrm{y}$ (range $21-30 \mathrm{y})$, and body mass index $(\mathrm{kg} /$ $\mathrm{m}^{2}$ ) of $23 \pm 1$ (range 18.1-27.9). Inclusion and exclusion criteria were as previously reported. ${ }^{2}$ The study complied with the Declaration of Helsinki and was approved by the ethical review board of the University of Fribourg; all participants gave written consent.

\subsection{Experimental design}

Prior to the day of testing, participants visited the laboratory to familiarize themselves with the experimental procedure and equipment. All participants were requested to avoid physical activity, caffeine, and dietary supplements for $24 \mathrm{~h}$ prior to testing. On the day of testing, participants arrived at 8 h00 following a $12 \mathrm{~h}$ overnight fast. After the participant voided his/her bladder, body weight and height were measured. EE and respiratory quotient (RQ) were measured using the Deltatrac II ventilated hood system (Datex-Ohmeda, Instrumentarium Corp, Helsinki, Finland) adapted for measurement in a variety of postures ${ }^{2}$ (Fig. 1).

Participants were seated comfortably in a car seat adapted for calorimetric monitoring, with metabolic measurement conducted until stabilization of EE for at least $15 \mathrm{~min}$, preceded by $30 \mathrm{~min}$ of rest. The ventilated hood was then removed and the participant asked to lie on a comfortable clinical table with their head resting on an ergonomic pillow, with as little exertion as possible. After transition and measurement stabilization, EE and RQ were recorded for $20 \mathrm{~min}$ in the supine position, followed by a second $30 \mathrm{~min}$ measurement in the seated position. The participant was instructed to relax and avoid movement, and in order to reduce boredom and accompanying stress, was allowed to watch a calm movie or a documentary throughout the metabolic measurements. In addition to calorimetric monitoring, heart rate (HR) and breathing (BR) were measured using a wireless physiological monitoring system (Equivital EQ-01, Hidalgo, Cambridgeshire, UK).

\subsection{Data and statistical analysis}

All data are presented as Mean \pm SEM unless otherwise stated. The statistical treatment of data was performed using the computer software STATISTIX 8 (Analytical Software, St. Paul, Minnesota, USA).

\section{Results}

There was no significant difference in EE between the supine and sitting measurements, nor between the two sitting measurements. Additionally, when separated by gender, no differences between supine and sitting EE were observed (Fig. 2A).

Furthermore, in a subgroup of subjects $(n=5)$ who repeated the experimental protocol on three different days, no differences in sitting versus supine REE were found on any day.

However, despite no change in EE, HR was found to be significantly higher whilst sitting than supine by 7 beats/min $(p<0.001)$, this difference was also observed when the data was separated according to gender (Fig. 2B). Similarly, the RQ was slightly, but significantly lower in the two sitting periods compared to the supine ( $p<0.005$; Fig. 2C). No change in BR was observed between the postures (Fig. 2D).

\section{Discussion}

With considerable discrepancies in reported effects of posture on energy expenditure, ${ }^{2,3,5}$ the present study sought to elucidate whether REE measured under standardized conditions in a comfortable seated posture differed to supine (BMR) measurements, using the ventilated hood system to measure EE in both postures.

Our results indicate that the REE after an overnight fast does not significantly differ during sitting compared to lying. This result is in agreement with the studies of both McCarthy ${ }^{3}$ and Levine ${ }^{4}$ where EE measured in a comfortable, motionless sitting position (with the back and legs supported) was not significantly different from EE measured in supine position. However, with concern that facemask or mouthpiece/nose clip systems may under- or overestimate EE, the present study is the first to be conducted using the ventilated hood system, the "method of choice" for the measurement of REE.

In addition to the indirect calorimetric method employed, another explanation for the discrepancies in the reported effects of
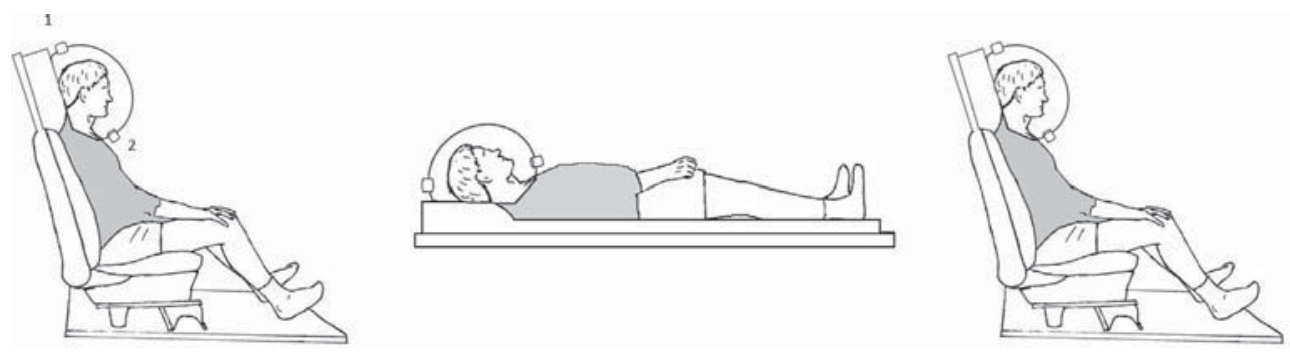

Fig. 1. Schema of experimental design. Posture-adapted ventilated hood indirect calorimetry set-up for sitting and supine measurements. The shaded area shows that the area of the subject covered by the veil of the ventilated hood. $1=$ air inlet; $2=$ air outlet to Deltatrac II. 
POOLED

A

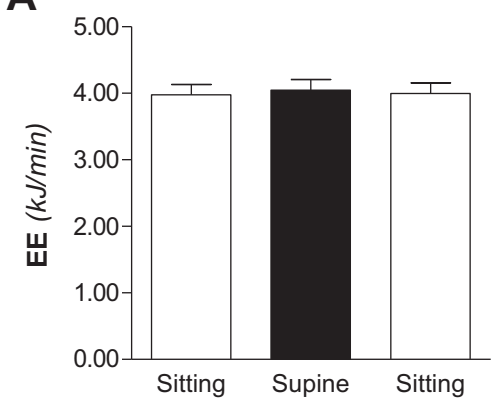

B

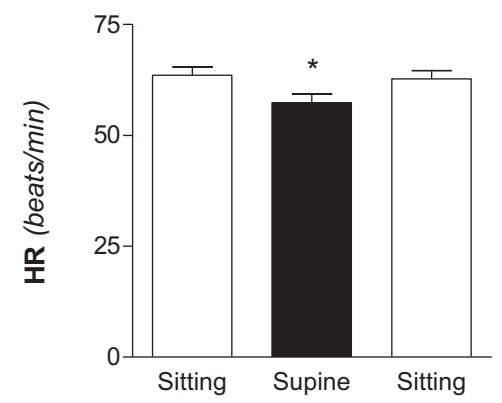

C

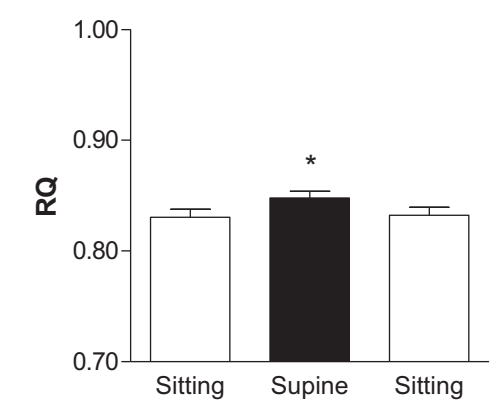

D

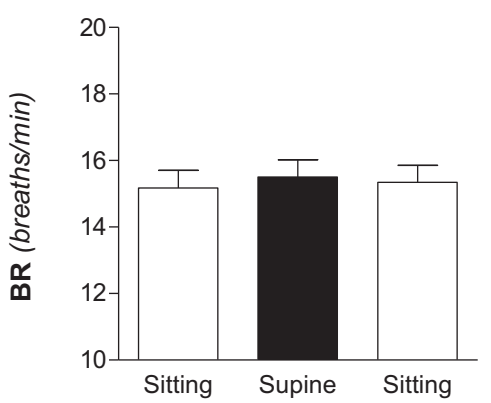

MEN

WOMEN
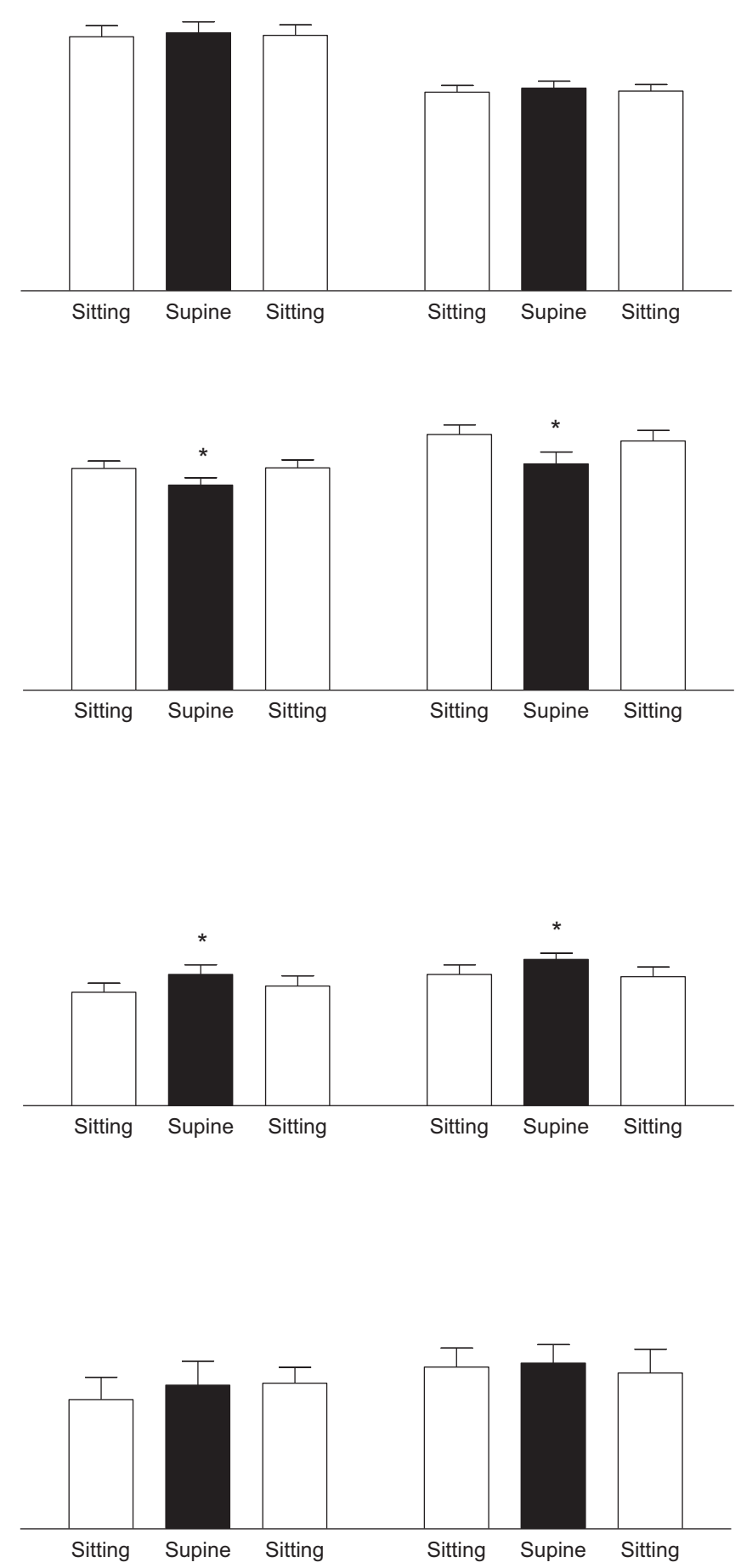

Fig. 2. Energy expenditure (EE in kJ/min; Panel A); heart rate (HR, beats/min; Panel B), respiratory quotient (RQ; Panel C), and breathing rate (BR, breaths/min; Panel D) separated by gender (pooled on left, men in centre, women on right) in sitting versus supine posture. All values were calculated as the mean ( \pm SEM) of the last $15-20$ min of steady state measurements in each posture. *Significantly different $(p<0.05)$ for other postures as assessed by repeated-measures ANOVA followed by Tukey's test.

posture on EE is the type of bed/seat used. For example, the energy cost of maintaining a posture on an exercise ball with no back support is likely to be higher ${ }^{8}$ compared to a comfortable seat where the back and legs are supported, as was used in our study.

In the present study, the higher value in HR found in the sitting position compared to lying down can be explained by the direct influence of gravity, with a vertical shift of blood below heart level and unloading of the baroreceptors. ${ }^{9}$ Furthermore, the supine position, with the legs at the same level as the heart, contributes to a facilitated venous return and a marked increase in heart volume due to displacement of blood volume from the extremities into the central circulation. This is in line with another study showing a $9 \%$ 
higher HR in the sitting than in the supine position. ${ }^{10}$ Nonetheless, the increase in HR during sitting versus lying is small ( +7 beats/ $\min$ ), and was not associated with a significant increase in REE.

Similarly, despite no differences in REE between the two postures, our results indicate a small but statistically lower RQ while sitting compared to lying. This decrease in RQ (reflecting an increase in fat relative to carbohydrate oxidation) during sitting may be attributed to the recruitment of postural muscle fibers, which are known to use primarily lipids as fuel.

In conclusion, this study suggests that the measurement of REE in a comfortable sitting position, using the ventilated hood system, under standardized conditions that include its measurements after an overnight fast, serves as a good proxy of BMR. It also underscores the applicability of the ventilated hood system to measurements of resting energy expenditure and substrate oxidation in the sitting posture, which, compared to the supine posture, may be more acceptable to the subject/patient participating in postprandial metabolic studies lasting several hours.

\section{Conflict of interest}

None.

\section{Acknowledgments}

The authors' contributions were as follows: JLM-C, DS, JPM YS and AGD designed research; JLM-C and DS conducted research;
JLM-C analyzed data and wrote the initial draft of the manuscript; AGD had primary responsibility for final content. All authors contributed towards, read and approved the final manuscript.

All authors declare that they have no conflict of interest. This study received no specific funding.

\section{References}

1. Wilmot EG, Edwardson CL, Achana FA, Davies MJ, Gorely T, Gray LJ, et al. Sedentary time in adults and the association with diabetes, cardiovascular disease and death: systematic review and meta-analysis. Diabetologia 2012;55: 2895-905.

2. Miles-Chan JL, Sarafian D, Montani JP, Schutz Y, Dulloo A. Heterogeneity in the energy cost of posture maintenance during standing relative to sitting: phenotyping according to magnitude and time-course. PLoS One 2013;8. e65827.

3. McCarthy RT. The metabolic cost of maintaining five fixed body positions. Nurs Res 1968:17:539-44.

4. Levine JA, Schleusner SJ, Jensen MD. Energy expenditure of nonexercise activity. Am J Clin Nutr 2000;72:1451-4.

5. Kanade AN, Gokhale MK, Rao S. Energy costs of standard activities among Indian adults. Eur J Clin Nutr 2001;55:708-13.

6. Forse RA. Comparison of gas exchange measurements with a mouthpiece, face mask, and ventilated canopy. JPEN J Parenter Enteral Nutr 1993;17:388-91.

7. Henry CJ. Basal metabolic rate studies in humans: measurement and development of new equations. Public Health Nutr 2005;8:1133-52.

8. Beers EA, Roemmich JN, Epstein LH, Horvath PJ. Increasing passive energy expenditure during clerical work. Eur J Appl Physiol 2008;103:353-60.

9. Singer W, OpferGgehrking TL, McPhee BR, Hilz MJ, Low PA. Influence of posture on the Valsalva manoeuvre. Clin Sci (Lond) 2001;100:433-40.

10. Jones AY, Dean E. Body position change and its effect on hemodynamic and metabolic status. Heart Lung 2004;33:281-90. 